\title{
Direct white noise characterization of short-channel MOSFETs
}

This paper was downloaded from TechRxiv (https://www.techrxiv.org).

\section{LICENSE}

CC BY 4.0

SUBMISSION DATE / POSTED DATE

03-12-2020 / 21-02-2021

CITATION

Ohmori, Kenji; Amakawa, Shuhei (2020): Direct white noise characterization of short-channel MOSFETs. TechRxiv. Preprint. https://doi.org/10.36227/techrxiv.13325237.v2

DOI

10.36227/techrxiv.13325237.v2 


\title{
Direct white noise characterization of short-channel MOSFETs
}

\author{
Kenji Ohmori, Member IEEE, and Shuhei Amakawa, Member, IEEE
}

\begin{abstract}
On-wafer evaluation of white thermal and shot noise in nanoscale MOSFETs is demonstrated by directly sensing the drain current under zero- and nonzerodrain-bias $\left(V_{d}\right)$ conditions for the first time, without recourse to a hot noise source, commonly needed in noise figure measurement. The dependence of white noise intensity on the drain bias clearly shows thermal noise at $V_{\mathrm{d}}=0 \mathrm{~V}$ and shot noise at $V_{\mathrm{d}}>0 \mathrm{~V}$ with its gate-biasdependent suppression. An empirical expression for the Fano factor (shot-noise suppression factor) that is wellbehaved even at $V_{\mathrm{d}}=0 \mathrm{~V}$ exactly and suitable for measurement-based evaluation is proposed. The direct measurement approach could allow more accurate and predictive noise modeling of RF MOSFETs than has conventionally been possible.
\end{abstract}

Index Terms-MOSFET, thermal noise, shot noise, 1/f noise, device modeling

\section{Introduction}

MOSFETs are known to exhibit flicker (or $1 / f$ ) noise at low frequencies (LF) and white noise at high frequencies (HF). Physical origins of flicker noise and white noise differ, and therefore, their power spectral densities are independent of each other. White noise can be further classified into thermal noise and shot noise, among others. Unlike the relationship between flicker noise and white noise, thermal noise and shot noise are not completely independent of each other [1-5].

Physics of noise in deep-submicrometer MOSFETs is much more involved than that in long-channel MOSFETs [611]. HF noise critically affects operation of very wideband RF circuits, especially millimeter-wave circuits, because the noise integrated over a wide bandwidth contributes to the signal-tonoise ratio (SNR) of a wireless system. Recently, adverse effects of white noise from a local oscillator on wideband millimeter-wave circuits have experimentally been confirmed $[12,13]$. Of course, phase noise of oscillators and frequency synthesizers below the white noise region, too, has always been and will increasingly be a concern in RF circuit design [14]. Accurate noise measurements of transistors over a wide frequency range, including the white noise region and the transition region to that region, therefore are essential not only for elucidating the physics behind but also for developing predictive device noise models for circuit design. Models built only from extrapolated data may well necessitate more silicon respins.

White noise in MOSFETs is typically characterized by noise figure (NF) measurement at RF, where flicker noise is negligible. In the Y-factor method of NF measurement, a known good "hot" white noise source is required to provide two ("hot" and "cold" (room temperature)) reference noise temperatures $[15,16]$. The hot or cold white noise is impressed to the device under test (DUT) during the measurement. The method is typically applicable down to $10 \mathrm{MHz}$ at best and usually requires a complicated de-embedding procedure, which adds to measurement uncertainty. LF (flicker) noise measurement, on the other hand, is performed by directly sensing noise generated by the DUT under "cold" dc-biased conditions. Several systems for LF noise measurement are commercially available. Although a typical maximum measurement frequency is a few tens of $\mathrm{MHz}$ in product specifications, in practice, frequency roll-off due to parasitic capacitance tends to make the maximum measurable frequency considerably lower, often below $1 \mathrm{MHz}$. Noise measurement around the $\mathrm{MHz}$ range is not very well covered by either method. Since typical MOSFET noise spectra become white only above $1 \mathrm{MHz}$ or higher, it is difficult to apply commercial LF noise measurement systems to white-noise measurement.

There have, nevertheless, been some reports on "cold" direct noise measurement of discrete MOSFETs over frequencies ranging from flicker noise region to white noise region $[17,18]$. We also chose a "cold" dc-biased approach for direct on-wafer device noise measurement. A proof-of-concept noise probe that extended the maximum measurable frequency to above $100 \mathrm{MHz}$ was demonstrated in [19,20]. It has a broadband low-noise amplifier (LNA) built in the probe itself, thereby reducing the parasitic capacitance significantly. At present, an improved version of the noise probe is commercially available [21]. Measurements can be conducted even at and near zero drain bias $\left(V_{\mathrm{d}}=0 \mathrm{~V}\right)$, which is not always straightforward by other means of measurement.

\section{MEASUREMENTS}

In this study, we demonstrate measurement of MOSFET white noise by utilizing a noise probe, shown in Fig. 1(a). Figure 1(b) shows a schematic diagram of the measurement system. The ac-component (i.e., noise) of dc drain current is amplified by the LNA in the noise probe and its output is read by a spectrum analyzer (N9030A, Agilent Technologies). Each individual noise probe is calibrated by extensive measurements, and that information is stored in a postprocessing software program [21]. It calculates the drain current noise from raw power spectrum reading by the spectrum analyzer, accounting for the noise generated by the LNA. The noise generated by the spectrum analyzer itself is accounted for, in effect, by turning on its Noise Floor Extension option [22]. Measurement results at low frequencies $(<100 \mathrm{kHz})$, where commercial LF 
measurement solutions work reliably, correlate well with results from such a system (9812D, ProPlus Design Solutions). In all measurements presented in the following, we employed a semiconductor device analyzer (B1500A, Agilent Technologies), equipped with four high-resolution sourcemeasure units (HR-SMUs), for biasing DUTs. Custom-built low-pass filters were used to filter out noise from the HR-SMUs.

We characterized N-MOSFETs with gate length/width of $120 \mathrm{~nm} / 10 \mu \mathrm{m}$, which were fabricated by using a $0.13-\mu \mathrm{m}$ CMOS technology. Each DUT has two sets of ground-signal-
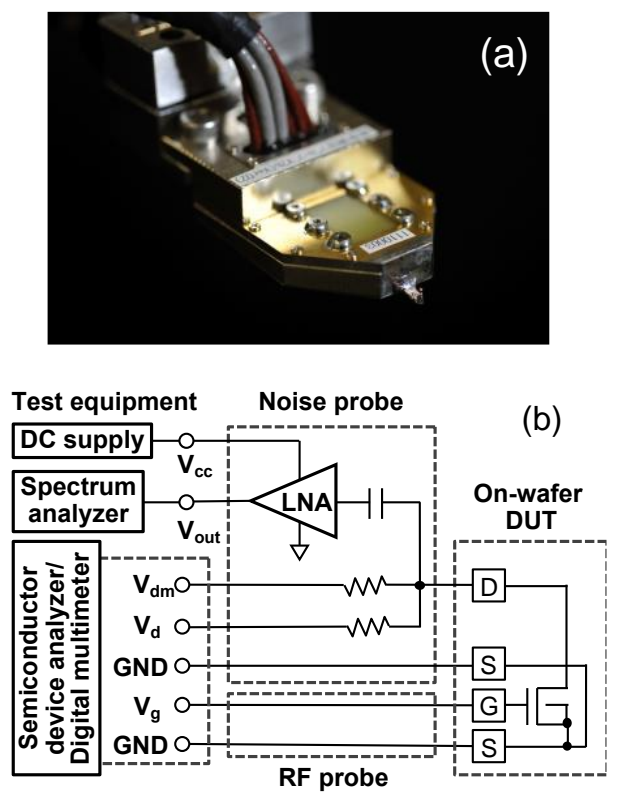

Figure 1 (a) A picture of a noise probe. An LNA is located inside the probe. (b) Schematic diagram of the measurement system. $V_{\mathrm{dm}}$ is for monitoring the drain voltage.

ground (GSG) pads: one connected to the drain and the source, and the other connected to the gate and the source. The gate was biased through an RF probe and the drain was biased through and probed by the noise probe. All measurements were conducted at room temperature between 24 and $26^{\circ} \mathrm{C}$.

\section{RESUltS AND DISCUSSION}

Figure 2 shows $I_{\mathrm{d}}-V_{\mathrm{d}}$ curves of an N-MOSFET at gate voltages, $V_{\mathrm{g}}$, of $0.5,0.6,0.7$, and $0.8 \mathrm{~V}$. The symbols on the curves represent bias points where noise measurements were carried out. The drain current increases even in the saturation region owing to the short channel length $(L=120 \mathrm{~nm})$. The threshold voltage estimated from an $I_{\mathrm{d}}-V_{\mathrm{g}}$ curve (not shown) at $V_{\mathrm{d}}$ of $30 \mathrm{mV}$ is $0.44 \mathrm{~V}$. Figure 3 (a) shows drain-current noise power spectral density, $S_{\text {Id }}$, for $V_{\mathrm{d}}=0 \mathrm{~V}$. The spiky peaks observed in the frequency range from 20 to $400 \mathrm{kHz}$ were induced by gate biasing. The white noise seen above $1 \mathrm{MHz}$ can be regarded as thermal noise associated with the differential resistance $R_{\text {dif }}=\partial V_{\mathrm{d}} / \partial I_{\mathrm{d}}$ [23]. As $V_{\mathrm{g}}$ increases, $R_{\text {dif }}$ decreases, resulting in higher white noise levels. We extracted $S_{\text {Id }}$ values at $500 \mathrm{MHz}$ and compared them with theoretical values in Fig. 3(b). The horizontal axis is the theoretical thermal current noise spectral density $S_{\text {th }}=4 k_{\mathrm{B}} T / R_{\text {dif }}$ at $V_{\mathrm{d}}=0 \mathrm{~V}$, where $k_{\mathrm{B}}$ and $T$ are the Boltzmann constant and the DUT absolute temperature, respectively. A very good agreement was obtained

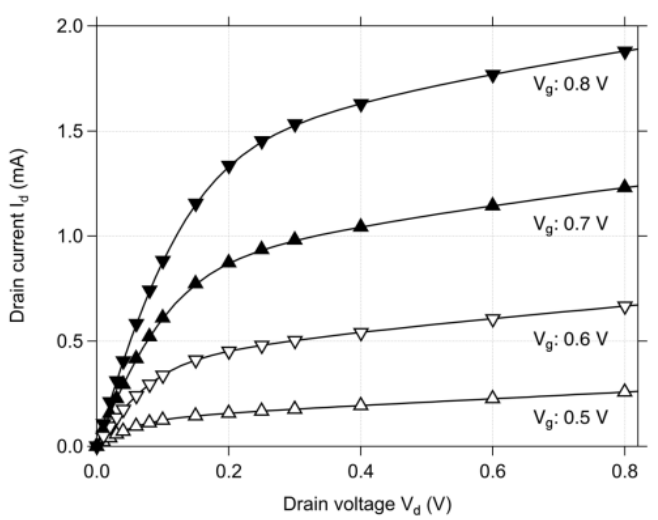

Figure 2 Measured $I_{\mathrm{d}}-V_{\mathrm{d}}$ curves of an N-MOSFET with gate length/width of $120 \mathrm{~nm} / 10 \mu \mathrm{m}$. The triangular symbols indicate bias points at which noise measurements were made.
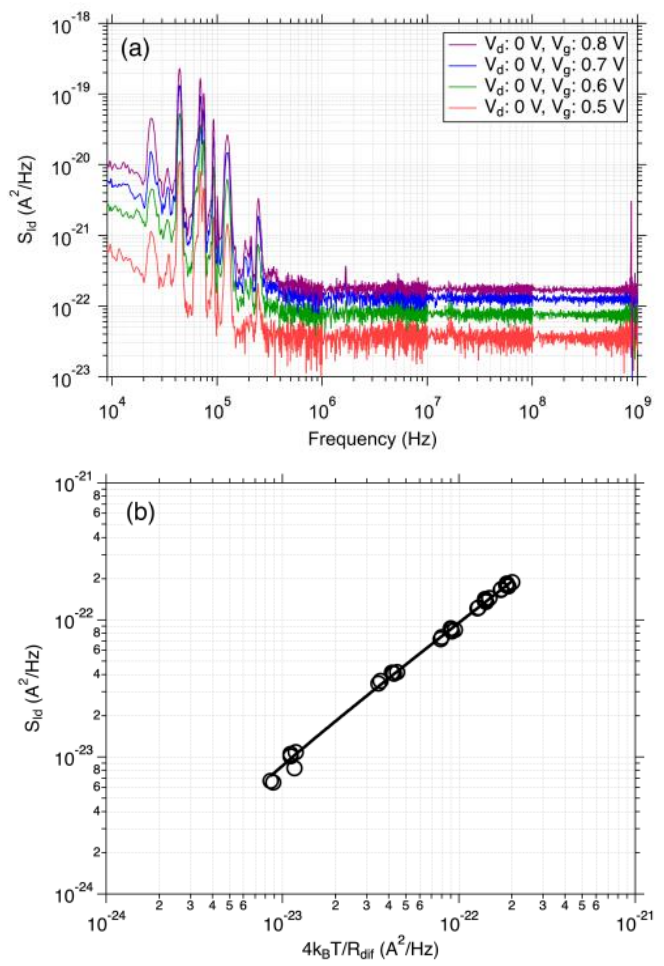

Figure 3 (a) Drain current noise spectral density $S_{\text {Id }}$ for $V_{\mathrm{g}}=$ $0.5,0.6,0.7,0.8 \mathrm{~V}$ and $V_{\mathrm{d}}=0 \mathrm{~V}$. (b) Measured $S_{\text {Id }}$ at $500 \mathrm{MHz}$ versus theoretical thermal noise $S_{\text {th }}=4 k_{\mathrm{B}} T / R_{\mathrm{dif}} . S_{\text {Id }}$ values from six DUTs are plotted.

from $7 \times 10^{-24}$ to $2 \times 10^{-22} \mathrm{~A}^{2} / \mathrm{Hz}$, corresponding to $R_{\mathrm{dif}}$ values from 1670 to $83.5 \Omega$, respectively.

When a nonzero $V_{\mathrm{d}}$ is applied, a dc drain current flows. Figure 4(a) shows the $S_{\text {Id }}$ spectra of six MOSFETs with the same dimensions under the same bias condition $\left(V_{\mathrm{d}}=0.2 \mathrm{~V}\right.$, $V_{\mathrm{g}}=0.7 \mathrm{~V}$ ). Flicker noise is considered to result from a large number of traps located near the channel [5]. Each trap exhibits a Lorentzian power spectrum with a certain time constant, also known as the burst noise [23]. In nanoscale MOSFETs, the number of traps in a device is small and some Lorentzian components become visible as in Fig. 4(a) [24,25]. Such outlying components manifest themselves in the time domain as random telegraph noise (RTN). By measuring many enough 

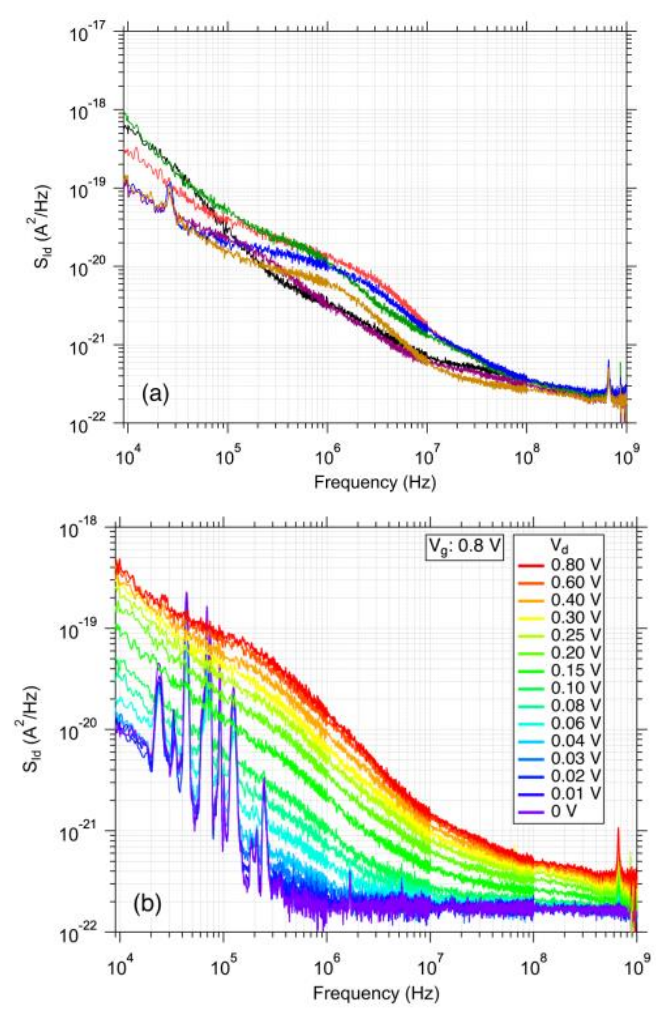

Figure 4 (a) Variability of noise spectral densities of six DUTs over a frequency range from $9 \mathrm{kHz}$ to $1 \mathrm{GHz}$. The bias condition was $V_{\mathrm{d}}=$ $0.2 \mathrm{~V}$ and $V_{\mathrm{g}}=0.7 \mathrm{~V}$. (b) Noise spectral densities for $V_{\mathrm{g}}=0.8 \mathrm{~V}$ and $V_{\mathrm{d}}$ ranging from 0 to $0.8 \mathrm{~V}$.
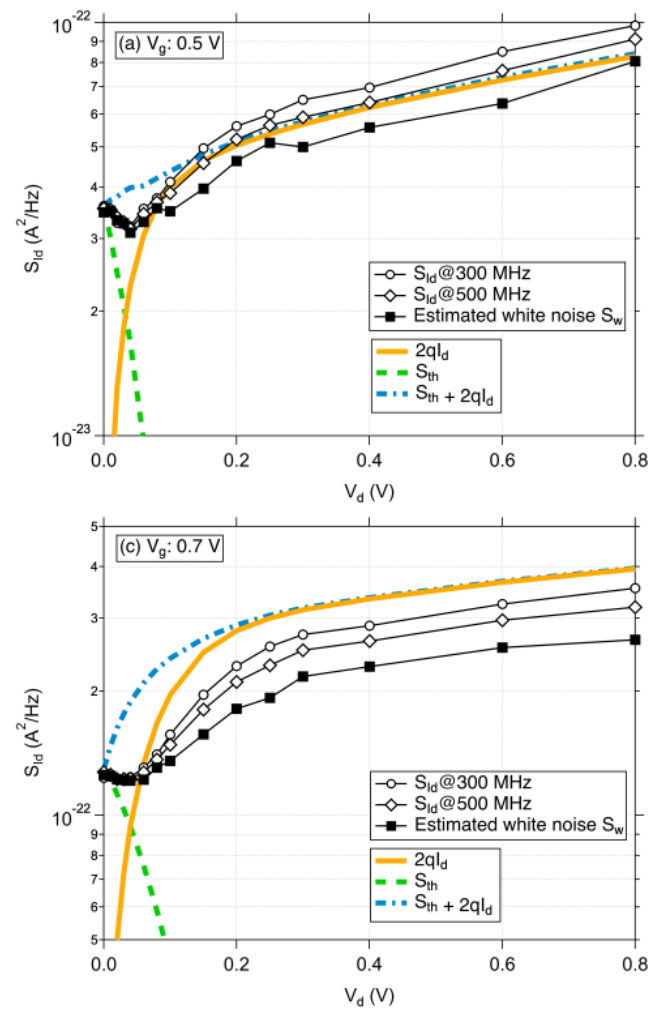

Figure 5 Dependence of noise power spectral density on $V_{\mathrm{d}}$ for $V_{\mathrm{g}}$ of (a) $0.5 \mathrm{~V}$, (b) $0.6 \mathrm{~V}$, (c) $0.7 \mathrm{~V}$, and (d) $0.8 \mathrm{~V}$. Open circles and diamonds show measured $S_{\mathrm{Id}}$ at 300 and $500 \mathrm{MHz}$, respectively. Solid squares show estimated white noise $S_{\mathrm{w}}$. Solid (orange) lines show full shot noise $2 q I_{\mathrm{d}}$. Dashed (green) lines show theoretical thermal noise $S_{\mathrm{th}}=4 k_{\mathrm{B}} T R_{\mathrm{ch}}\left(V_{\mathrm{d}}\right) /\left[R_{\mathrm{dif}}\left(V_{\mathrm{d}}\right)\right]^{2}$. Dot-dashed (blue) lines show the sum $2 q I_{\mathrm{d}}+S_{\mathrm{th}}$. devices in the time domain and analyzing the results statistically, further information could be obtained about traps near the channel $[26,27]$. Studies on RTN have generally been performed in a LF region due to measurement limitations. Since the raw output from the noise probe is a time-domain waveform, the use of a real-time oscilloscope in place of a spectrum analyzer (Fig. 1(b)) will allow us to observe RTN beyond $1 \mathrm{MHz}$. In this study, we focus on frequency-domain evaluation of white noise. Note that Lorentzian components below $10 \mathrm{MHz}$ do not usually affect the shape of $S_{\text {Id }}(f)$ above $100 \mathrm{MHz}$ because each of them drops with $1 / f^{2}$. Therefore, $S_{\text {Id }}(f)$ above $100 \mathrm{MHz}$ can be regarded as the sum of white noise and residual flicker noise. The noise spectral density above $100 \mathrm{MHz}$ can, therefore, be approximated as

$$
S_{\text {Id }}(f)=A / f^{\beta}+S_{\mathrm{w}}
$$

where $-\beta(\approx-1)$ is the slope of flicker noise power spectrum on a log-log plot, $S_{\mathrm{w}}$ is the white noise level, and $\left(A / S_{\mathrm{w}}\right)^{1 / \beta}$ is the corner frequency (onset frequency of the white noise region).

Figure 4(b) shows $S_{\mathrm{Id}}(f)$ at $V_{\mathrm{g}}=0.8 \mathrm{~V}$ with $V_{\mathrm{d}}$ ranging from 0 to $0.8 \mathrm{~V}$. It clearly shows that predominant noise changes from flicker noise $\left(1 / f^{\beta}\right)$ to white noise $\left(1 / f^{0}\right)$ as frequency becomes higher. As $V_{\mathrm{d}}$ becomes larger, flicker noise power increases, resulting in a higher corner frequency. Although the slope of $S_{\text {Id }}(f)$ becomes very small at high frequencies $\left(>100 \mathrm{MHz}\right.$ ), the slope for large $V_{\mathrm{d}}$ values is not quite zero. Note that the absolute value of the slope of (1) above the corner frequency is smaller than $\beta(\approx 1)$.
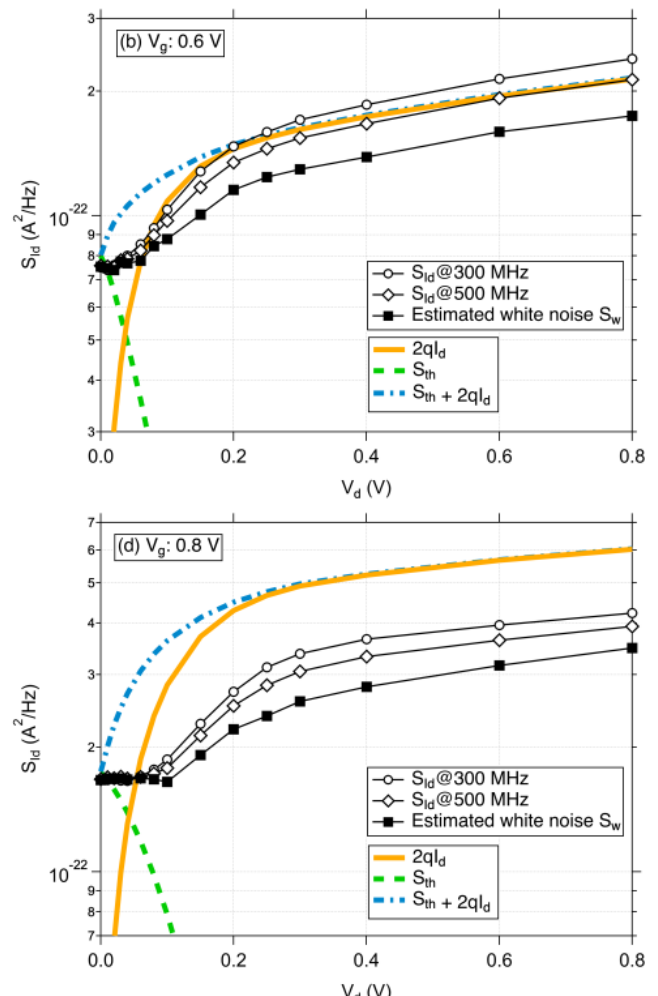
Figure 5 shows dependence of $S_{\mathrm{Id}}$ on $V_{\mathrm{d}}$ and $V_{\mathrm{g}}$. Open circles and diamonds show measured $S_{\mathrm{Id}}$ at 300 and $500 \mathrm{MHz}$, respectively. As seen in Fig. 4(b), these values are nearly the same in the low- $V_{\mathrm{d}}$ region $\left(V_{\mathrm{d}}<0.1 \mathrm{~V}\right)$. Meanwhile, as $V_{\mathrm{d}}$ increases, $S_{\mathrm{Id}}$ at $500 \mathrm{MHz}$ becomes lower than that at $300 \mathrm{MHz}$ due to higher levels of residual flicker noise (Fig. 4(b)). The solid squares in Fig. 5 show estimated white noise $S_{\mathrm{w}}$, calculated by using (1) with $\beta=1$ and measured values of $S_{\text {Id }}$ at 300 and $500 \mathrm{MHz}$, from which $A$, too, can be determined. Using measured $I_{\mathrm{d}}-V_{\mathrm{d}}$ curves (Fig. 2), we calculated the full shot noise intensity, $2 q I_{\mathrm{d}}$, shown by the thick solid (orange) lines in Fig. 5. Theoretical $V_{\mathrm{d}}$-dependent thermal noise intensity is shown in Fig. 5 by the dashed (green) lines, given by $[23,28]$

$$
S_{\mathrm{th}}=\frac{4 k_{\mathrm{B}} T R_{\mathrm{ch}}\left(V_{\mathrm{d}}\right)}{\left[R_{\mathrm{dif}}\left(V_{\mathrm{d}}\right)\right]^{2}}
$$

where $R_{\mathrm{ch}}\left(V_{\mathrm{d}}\right)=V_{\mathrm{d}} / I_{\mathrm{d}}$ is the chord resistance [29]. Note that $R_{\text {ch }}\left(V_{\mathrm{d}}\right) \neq R_{\mathrm{dif}}\left(V_{\mathrm{d}}\right)$ unless $V_{\mathrm{d}}=0 \mathrm{~V}$. Note also that we used (2) instead of the better-known integral expression [10,23], because the latter requires more a priori knowledge about the DUT, making its purely measurement-based evaluation difficult.

The $S_{\mathrm{w}}$ values at $V_{\mathrm{d}}=0 \mathrm{~V}$, shown in Fig. 5, agree well with $S_{\text {th }}$, consistent with Fig. 3(b). Given the fact that experimentally observed shot noise is usually lower than the full shot noise [30], $S_{\mathrm{w}}$ should lie somewhere between $S_{\mathrm{th}}$ and $S_{\mathrm{th}}+2 q I_{\mathrm{d}}$. The latter is shown in Fig. 5 by the dot-dashed (blue) lines. As $V_{\mathrm{d}}$ increases, $S_{\mathrm{w}}$ increases similarly to $2 q I_{\mathrm{d}}$. The $V_{\mathrm{d}}$ value at the point of intersection of $2 q I_{\mathrm{d}}$ and $S_{\mathrm{th}}$ could be regarded as the onset point of shot noise dominance. Notably, $S_{\mathrm{w}}$ of Fig. 5(a) $\left(V_{\mathrm{g}}=0.5 \mathrm{~V}\right.$, moderate inversion), assumes a minimum value at $V_{\mathrm{d}} \approx 0.04 \mathrm{~V}$. Although the difference between the observed minimum and the $S_{\mathrm{w}}$ value at $V_{\mathrm{d}}=0 \mathrm{~V}$ is small, most of our DUTs showed similar behavior at $V_{\mathrm{g}} \lesssim$ $0.5 \mathrm{~V}$. It is known that, in weak inversion of a long-channel device, the following white noise power expression (3) can be derived whether assuming a thermal noise origin or a shot noise origin $[4,5]$.

$$
S_{\mathrm{w}, \mathrm{wi}}=2 q I_{\mathrm{sat}}\left(1+\mathrm{e}^{-q V_{\mathrm{d}} / k_{\mathrm{B}} T}\right),
$$

where $I_{\text {sat }}$ is the saturation current corresponding to the given gate voltage. (3) implies that at $V_{\mathrm{d}}=0 \mathrm{~V}, S_{\mathrm{w}, \mathrm{wi}}=4 q I_{\mathrm{sat}}=S_{\mathrm{th}}$. Although our devices are short-channel and we have no weakinversion data because of the limited device width of $W=$ $10 \mu \mathrm{m}$, the observed minimum in $S_{\mathrm{w}}$ at $V_{\mathrm{d}} \approx 0.04 \mathrm{~V}$ could be related to the second term of (3), originating from electrons flowing in the reverse direction (from drain to source). Theoretically expected decrease in $S_{\mathrm{w}}$ (versus $V_{\mathrm{d}}$ ) in strong inversion due to channel pinch-off [5] is not clearly observed in Fig. 5(d).

Fano considered a theoretical limit for the statistical fluctuation in the number of traveling particles [31]. The full shot noise corresponds to the situation where statistical fluctuation is maximized. The so-called Fano factor $F$ ( $\leq$ 1) [32] is a coefficient for shot noise suppression and is defined as the ratio of the actual shot noise power to the full shot noise power $2 q I_{\mathrm{d}} . F$ has successfully been employed for describing white noise of nanoscale MOSFETs when $V_{\mathrm{d}}$ is sufficiently high [30]. However, the conventional expression, $F=S_{\text {Id }} /$ $\left[2 q I_{\mathrm{d}} \operatorname{coth}\left(V_{\mathrm{d}} / 2 k_{\mathrm{B}} T\right)\right]$ [30], is not suitable for measurementbased evaluation of $F$ near $V_{\mathrm{d}}=0 \mathrm{~V}$. To find an expression suitable for measurement-based evaluation, it should be defined such that it equals unity at $V_{\mathrm{d}}=0 \mathrm{~V}$ in weak inversion, considering (3), which includes shot noise contributions from currents flowing in both directions [4], and Fig. 3(b). We herein introduce an empirical expression for the Fano factor $F$ that exhibits the desired behavior, as follows:

$$
F=\frac{S_{\mathrm{w}}}{2 q I_{\mathrm{d}}+S_{\mathrm{th}}}
$$

where $I_{\mathrm{d}}$ is the measured drain current, $S_{\text {th }}$ is given by (2), and $S_{\mathrm{w}}$ is the measured (estimated) white noise. The denominator of (4) corresponds to the dot-dashed (blue) lines in Fig. 5. The $S_{\text {th }}$ in the denominator makes up for the "lost" full shot noise when $V_{\mathrm{d}}$ is below a few times the thermal voltage, $k_{\mathrm{B}} T / q \approx$ $0.026 \mathrm{~V}$.

Figure 6 shows the suppression factor $F$ as a function of $V_{\mathrm{d}}$ for $V_{\mathrm{g}}=0.5,0.6,0.7$, and $0.8 \mathrm{~V}$. $F$ decreases as $V_{\mathrm{g}}$ increases presumably due to the reduction of the potential barrier near the source [33]. Shot noise is generally considered to be generated primarily due to the presence of this potential barrier, although quasi-ballistic transport in the pinched-off region near the drain may also play a role in short-channel devices. More precise discussion will require physical modeling and simulation including quasi-ballistic transport and Coulomb interaction [6,34].

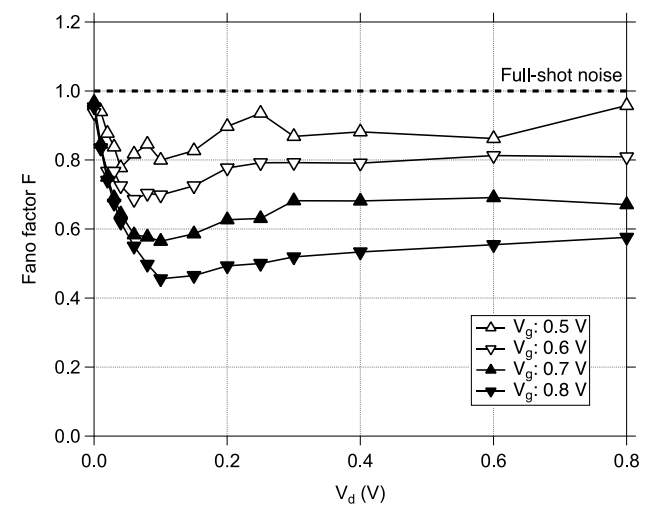

Figure 6 Fano factor $F$ versus drain voltage $V_{\mathrm{d}}$ for $V_{\mathrm{g}}=$ $0.5,0.6,0.7$, and $0.8 \mathrm{~V}$.

\section{CONCLUSIONS}

In summary, we have developed a methodology for conveniently characterizing white noise of dc-biased nanoscale MOSFETs through wafer probing without a hot noise source or complicated de-embedding procedures. Using the approach developed, we have successfully measured white noise in shortchannel MOSFETs under various bias conditions, including $V_{\mathrm{d}}=0 \mathrm{~V}$. We also proposed an empirical expression for the Fano factor (shot-noise suppression factor) $F$, which is wellbehaved at $V_{\mathrm{d}} \rightarrow 0 \mathrm{~V}$ and suitable for purely measurementbased, modeling-free evaluation.

In general, various factors such as device structures and 
dimensions, impurity profiles, and choice of materials should affect $F$ and other properties. Therefore, actual measurement of devices will be important for understanding of device properties and physics.

Note also that in circuits like an FET resistive mixer, which boasts the best low-noise performance among FET mixers [35], FETs are used under a zero-drain-bias condition. Accurate noise modeling, including near $V_{\mathrm{d}}=0 \mathrm{~V}$, therefore is very important. Actual measurement of white noise will be essential for developing a predictive transistor noise model valid in all regions of operation. The direct measurement approach could be a perfect complement to NF measurement and device modeling based on the latter measurement method [36].

\section{ACKNOWLEDGMENT}

KO would like to thank Prof. N. Sano of University of Tsukuba for critical comments on an early version of the manuscript.

\section{REFERENCES}

[1] J. B. Johnson, "Thermal agitation of electricity in conductors," Phys. Rev., vol. 32, no. 1, pp. 97-109, July 1928.

[2] H. Nyquist, "Thermal agitation of electric charge in conductors," Phys. Rev., vol. 32, no. 1, pp. 110-113, July 1928.

[3] R. Landauer, "Solid-state shot noise," Phys. Rev. B, vol. 47, pp. 16427-16432, June 1993.

[4] R. Sarpeshkar, T. Delbrück, and C. A. Mead, "White noise in MOS transistors and resistors," IEEE Circuits Devices Mag., vol. 9, no. 6, pp. 23-29, Nov. 1993.

[5] Y. Tsividis and C. McAndrew, Operation and Modeling of the MOS Transistor, $3^{\text {rd }}$ ed., Oxford, UK: Oxford University Press, 2011.

[6] N. Sano, K. Natori, K. Matsuzawa, and M. Mukai, "Current fluctuation characteristic of sub-0.1 micron device structures: a Monte Carlo study,” Jpn. J. Appl. Phys., vol. 38, no. 5A, pp. L531-533, May 1999.

[7] J. C. J. Paasschens, A. J. Scholten, and R. van Langevelde, "Generalizations of the Klaassen-Prins equation for calculating the noise of semiconductor devices," IEEE Trans. Electron Devices, vol. 52, no. 11, pp. 2463-2472, Nov. 2005.

[8] R. Navid, C. Jungemann, T. H. Lee and R. W. Dutton, "High-frequency noise in nanoscale metal oxide semiconductor field effect transistors," J. Appl. Phys., vol. 101, no. 12, 124501, June 2007.

[9] V. M. Mahajan, P. R. Patalay, R. P. Jindal, H. Shichijo, S. Martin, F.-C. Hou, C. Machala, and D. E. Trombley, "A physical understanding of RF noise in bulk nMOSFETs with channel lengths in the nanometer regime," IEEE Trans. Electron Devices, vol. 59, no. 1, pp. 197-205, Jan. 2012.

[10]G. D. J. Smit, A. J. Scholten, R. M. T. Pijper, L. F. Tiemeijer, R. van der Toorn, and D. B. M. Klaassen, "RF-noise modeling in advanced CMOS technologies," IEEE Trans. Electron Devices, vol. 61, no. 2, pp. 245-254, Feb. 2014.

[11]A. J. Scholten, D. G. J. Smit, R. M. T. Pijper, and L. F. Tiemeijer, "Benchmark tests for MOSFET thermal noise models," in Noise in Nanoscale Semiconductor Devices, Cham, Switzerland: Springer Nature Switzerland AG, 2020, pp. 687-710.

[12]J. Chen, Z. S. He, D. Kuylenstierna, T. Eriksson, M. Hörberg, T. Emanuelsson, T. Swahn, and H. Zirath, "Does LO noise floor limit performance in multi-gigabit millimeter-wave communication," IEEE Microwave Compon. Lett., vol. 27, no. 8, pp. 769-771, Aug. 2017.

[13]J. Chen, D. Kuylenstierna, S. E. Gunnarsson, Z. S. He, T. Eriksson, T. Swahn, and H. Zirath, "Influence of white LO noise on wideband communication," IEEE Trans. Microwave Theory Tech., vol. 66, no. 7, pp. 3349-3359, July 2018.

[14]E. Rubiola, Phase Noise and Frequency Stability in Oscillators,
Cambridge, UK: Cambridge University of Press, 2009.

[15]L. F. Tiemeijer, R. J. Havens, R. de Kort, and A. J. Scholten, "Improved Y-factor method for wide-band on-wafer noise-parameter measurements," IEEE Trans. Microwave Theory Tech., vol. 53, no. 9, pp. 2917-2925, Sep. 2005.

[16]Keysight Technologies, "Fundamentals of RF and microwave noise figure measurements," Application Note, 5952-8255E, 2019.

[17]A. A. Abidi, "High-frequency noise measurements on FETs with small dimensions," IEEE Trans. Electron Devices, vol. 33, no. 11, pp. 18011805, Nov. 1986.

[18]M. Manghisoni, L. Ratti, V. Re, and V. Speziali, "Instrumentation for noise measurements on CMOS transistors for fast detector preamplifiers," IEEE Trans. Nuclear Science, vol. 49, no. 3, pp. 12811286, June 2002.

[19]K. Ohmori, R. Hasunuma, W. Feng, and K. Yamada, "Continuous characterization of MOSFET from low-frequency noise to thermal noise using a novel measurement system up to $100 \mathrm{MHz}$," in Proc. Symp. VLSI Technol., 2012, pp. 143-144.

[20]K. Ohmori, R. Hasunuma, S. Yamamoto, Y. Tamura, H. Jiang, N. Ishihara, K. Masu, and K. Yamada, "Application of low-noise TIA ICs for novel sensing of MOSFET noise up to the GHz region," in Proc. Symp. VLSI Circuts, 2013, pp. C40-41.

[21]Entrope ${ }^{\circledR}$ Noise Probe, http://www.devicelab.co.jp

[22] Keysight Technologies, "Using Noise Floor Extension in an X-series signal analyzer," Application Note, 5990-5340EN, 2019.

[23]A. van der Ziel, Noise in Solid State Devices and Circuits. New York, NY, USA: John Wiley \& Sons, Inc., 1986.

[24]W. Feng, R. Hettiarachchi, Y. Lee, S. Sato, K. Kakushima, M. Sato, K. Fukuda, M. Niwa, K. Yamabe, K. Shiraishi, H. Iwai, and K. Ohmori, "Fundamental origin of excellent low-noise property in 3D SiMOSFETs $\sim$ Impact of charge-centroid in the channel due to quantum effect on 1/f noise ," in IEDM Tech. Dig., Dec. 2011, pp. 630-633.

[25]P. Srinivasan and S. Dey, "New and critical aspects of $1 / \mathrm{f}$ noise variability in advanced CMOS SoC technologies," in IEDM Tech. Dig., Dec. 2012, pp. 458-461.

[26]T. Nagumo, K. Takeuchi, S. Yokogawa, K. Imai, and Y. Hayashi, "New analysis methods for comprehensive understanding of random telegraph noise," in IEDM Tech. Dig., Dec. 2009, pp. 759-762.

[27]T. Nagumo, K. Takeuchi, T. Hase, and Y. Hayashi, "Statistical characterization of trap position, energy, amplitude and time constants by RTN measurement of multiple individual traps," in IEDM Tech. Dig., Dec. 2010, pp. 628-631.

[28]S. T. Hsu, A. van der Ziel, and E. R. Chenette, "Noise in space-chargelimited solid-state devices," Solid-State Electron., vol. 10, no. 2, pp. 129-135, Feb. 1967.

[29]L. Chua, "Resistance switching memories are memristors," Appl. Phys. $A$, vol. 102, pp. 765-783, Mar. 2011.

[30]X. Chen, C. H. Chen, and R Lee, "Fast evaluation of the highfrequency channel noise in nanoscale MOSFETs," IEEE Trans. Electron Devices, vol. 65, no. 4, pp. 1502-1509, Apr. 2018.

[31]U. Fano, "Ionization yield of radiations. II. The fluctuations of the number of ions," Phys. Rev., vol. 72, no. 1, pp. 26-29, July 1947.

[32]C. Beenakker and C. Schönenberger, "Quantum shot noise," Phys. Today, vol. 56, no. 5, pp. 37-42, May 2003.

[33]A. Rahman, J. Guo, S. Datta, and M. S. Lundstrom, "Theory of ballistic nanotransistors," IEEE Trans. Electron Devices, vol. 50, no. 9, pp. 1853-1864, Sep. 2003.

[34] G. Iannaccone, A. Betti, G. Fiori, "Suppressed and enhanced shot noise in one dimensional field-effect transistors," J. Comput. Electron., vol. 14, pp. 94-104, Feb. 2015.

[35]S. A. Maas, The RF and Microwave Circuit Design Cookbook, Artech House, Norwood, Massachusetts, 1998.

[36]I. Myeong, J. Kim, H. Ko, I. Song, Y. Kim, and H. Shin, “A simple and accurate modeling method of channel thermal noise using BSIM4 noise models," IEEE Trans. Comput.-Aided Des. Integr. Circuits Syst., vol. 39, no. 12, pp. 4351-4358, Dec. 2020. 